Ann. Zootech., I969, 18 (2), 95-Io6.

$$
\begin{aligned}
& \text { I.N.R.A. } 35905 \\
& \text { BIBLIOTHEQUE UO CROUELLE } \\
& \text { DOMAINE DE } 3039 \\
& \text { CLERMONT-FD CEDEX } 2
\end{aligned}
$$

\title{
EFFETS DE L'INTRODUCTION DE BÉLIERS VASECTOMISÉS DANS UN TROUPEAU MÉRINOS D'ARLES, QUINZE JOURS AVANT LE DÉBUT DE LA LUTTE DE PRINTEMPS, SUR L'APPARITION DES CESTRUS, LA FRÉQUENCE DES ERREURS DE DÉTECTION DES RUTS ET LA FERTILITÉ DES BREBIS
}

\author{
M. PRLD'LION et I. DENOY \\ Avec la collaboration technique de R. Devillard et C. SICARD \\ I.aboratoire de Zootechnie, \\ Centre de Recherches de Montpellier, \\ École nationale supérieure agronomique, 34 -Montpellier \\ Institut national de la Recherche agronomique
}

SOMMAIRE

L'étude de l'apparition des nestrus des brebis Mérinos d'Arles du domaine du Merle (PRud'Hon et al., I 966) ayant mis en évidence l'existenct, au printemps, d'un effet de la présence des béliers sur le déclenchement des cestrus des brebis, nous avons recherché, de 1965 à 1967, si cet effet se manifestait également lorsque l'introduction des béliers était avancée de i 5 jours.

Les résultats obtenus mettent en évidence un groupage des ostrus des brebis non allaitantes I 7 ct 23 jours après l'introduction des béliers dans le troupeau alors que chez les femelles allaitantes, la fréquence maximale des ostrus se situe 23 jours après la mise en place des boute-en-train. Ce groupage est plus marqué que lors des observations précédentes.

Les erreurs de détection des ruts au cours de la période de lutte contrôlée ont été sensiblement réduites, notamment chez les antenaises.

La fertilité des brebis au cours des 30 premiers jours de lutte a été améliorée en raison de l'élévation des taux d'xestrus et de grestation des antenaises et des brebis allaitantes.

\section{IN'TRODUC'TION}

L’influence de la présence des mâles sur l'activité ovarienne des femelles a été mise en évidence dans plusieurs espèces : la Souris (WhitTen, I956; LAamond, I959), le Rat (Hughes, I964), la Chèvre (Shelton, I960) ; dans l'espèce ovine cet 
effet psychique est particulièrement net au début de la saison sexuelle (UNDERWOOD et al., I944; Sinclair, I950 ; Schinckei,. I954; Eyar, I958; F,dgar et Bilker, I963; Prun'hon et al., ig66) ainsi qu'à la fin (Lishman et Hunter, ig66). Dans les races qui, comme le Mérinos, ont un repos sexuel saisonnier peu marqué, 1'introduction de béliers boute-en-train dans un troupeau, à n'importe quelle période de cet ancestrus, augmente le pourcentage de femelles extériorisant un comportement d'oestrus (RICHEs et WATSON, I954). Ein France, nous manquons de renseignements sur le comportement de nos principales races mais les observations effectuées en race Mérinos d'Arles (DAuzier et DENOY, I964; PrUD'HON et al., I966) ont montré l'influence très marquée de l'introduction des béliers sur l'apparition du comportement d'œstrus des brebis au moment de la lutte de printemps qui a lieu du Io mai au I5 juin. A cette époque, les chaleurs ne sont pas réparties également au cours des I7 premiers jours de lutte contrôlée, comme c'est le cas à l'automne, mais ont une distribution bimodale; les modes sont situés respectivement les $\mathrm{I}_{4}^{\mathrm{e}}$ et $2 \mathrm{O}^{\mathrm{e}}$ jours de lutte chez les brebis adultes, les $I 7^{\mathrm{e}}$ et $23^{\mathrm{e}}$ jours che $z$ les antenaises, les $26^{\mathrm{e}}$ et $35^{\mathrm{e}}$ jours chez les tardonnières (brebis ayant mis bas depuis 2 mois, en cours d'allaitement au moment de la lutte). Iu fait de la date relativement tardive d'apparition des ostrus et de la courte durée de la lutte, un certain nombre de femelles, non gestantes après saillie au premier cstrus, n'ont pas la possibilité d'être accouplées à nouveau et la fertilité du troupeau en est réduite.

En introduisant des béliers vasectomisés dans le troupeau le 26 avril, 15 jours avant que la lutte ne commence, nous nous sommes proposé de savoir si l'effet de la présence des mâles se manifestait déjà à cette époque et, dans ce cas, si la majeure partie des œestrus étaient groupés au cours des Io premiers jours de lutte.

Nous faisons le point, ici, des résultats obtenus pendant trois années de cette pratique en insistant notamment sur la distribution des fréquences d'apparition des astrus, le pourcentage d'anomalies de détection des ruts et la fertilité du troupeau après 30 jours de lutte. Ces résultats sont comparés à ceux que nous avons déjà obtenus de I959 à Ig64 (PRUD'HON et al., I966), lorsque les béliers étaient introduits seulement le premier jour de la lutte.

\section{MATÉRIEL ET MÉTHODES}

Cette étude porte sur l'apparition des ostrus de 8 o 8 brebis Mérinos d'Arles soumises à une lutte de printemps, au domaine du Merle, pendant la période I959-1967.

Les caractéristiques de l'élevage, les techniques de détection des ruts ainsi que les résultats enregistrés de I959 à I 964 ont déjà été décrits (PRUD'Hon et $a l$., 1966).

Au cours de ces six années, des béliers vasectomisés munis de harnais marqueurs étaient introduits dans le troupeau le premier jour de la lutte de printemps ( 22 mai \pm 2 jours) à raison d'un bélier pour 60 brebis environ; les brebis ainsi détectées, triées trois fois par jour, étaient placées dans les cases des béliers entiers destinés à les saillir. Après 3 ou 4 jours de présence dans ces box, elles étaient regroupées dans un troupeau de femelles saillies où d'autres boute-en-train assuraient le contrôle des nouveaux ostrus.

De 1965 à 1967 , les techniques de détection des ruts sont restées les mêmes mais les béliers vasectomisés ont été introduits dans le troupeau, sans harnais, I 5 jours avant le début de la lutte à raison d'un bélier pour 80 à Ioo brebis; ils ont été harnachés le premier jour de la lutte et leur nombre porté à I pour 60 à 80 brebis. 
Les effectifs d'antenaises et d'adultes soumis à la lutte de printemps au cours de ces deux périodes figurent au tableau $\mathrm{x}$.

TABLEAU I

Effectifs soumis à la lutte de printemps

\begin{tabular}{c|c|c|c|c}
\hline \hline Lot & Période & Antenaises & Adultes & Tardonnières (1) \\
\hline $\mathrm{J} \mathrm{0}$ & $1959-1964$ & 1283 & 3269 & 382 \\
\hline $\mathrm{J}(-15)$ & $1965-1967$ & 554 & $214_{4}$ & 333 \\
\hline
\end{tabular}

(I) Brebis ayant mis bas au mois de mars, soumises à la lutte de printemps au mois de mai suivant.

L'étude des anomalies de détection des ruts a été menée en tenant compte des critères déjà définis (PRUD'HON et al., I966); les durées de cycle inférieures à 15 jours ou supérieures à i 9 jours ont été considérées comme anormales; la signification statistique des différences de fréquence des erreurs a été étudiée à l'aide du test $\chi^{2}$ de Pearson.

Les termes : taux d'oestrus, taux de gestation et taux de fertilité utilisés dans cette étude ont déjà été définis précédemment (PRUD'HON et al., 1968).

Nous avons appelé lot J ( $\left.-\mathrm{r}_{5}\right)$ et lot Jo les lots de brebis au sein desquels les béliers vasectomisés ont été introduits respectivement 15 jours avant la lutte et le premier jour de la lutte.

Nous appelons tardonnières des brebis qui, ayant mis bas en février-mars, sont à nouveau soumises à la lutte de printemps.

\section{RÉSULTATS}

\section{A. - Apparition des cestrus}

Les courbes de fréquence d'apparition des premiers ruts des antenaises et des brebis adultes (fig. I) permettent les constatations suivantes :

- quelle que soit la date d'introduction des béliers, ces courbes présentent deux pointes principales séparées par un intervalle de 5 à 6 jours.

- dans le lot $\mathrm{J}\left(-\mathrm{I}_{5}\right)$ ces pointes sont très marquées, d'amplitude égale et situées le $2^{\mathbf{e}}$ et $1 \mathrm{e} 8^{\mathrm{e}}$ jours de lutte chez les antenaises, le $2^{\mathrm{e}}$ et le $7^{\mathrm{e}}$ jours de lutte chez les adultes. Elles sont donc parfaitement synchronisées et surviennent I7 et 23 à 24 jours après la mise en place des béliers boute-en-train ; de plus, une troisième pointe d'amplitude beaucoup plus faible apparaît les $I 4^{\mathrm{e}}, \mathrm{I} 5^{\mathrm{e}}$ et $\mathrm{I} 6^{\mathrm{e}}$ jours de lutte sur la courbe des brebis adultes;

- dans le lot Jo, les deux pointes ont une amplitude plus faible, une importance inégale chez les adultes, égale chez les antenaises. Elles ne sont pas synchronisées étant situées en moyenne $1 \mathrm{e} \mathrm{I}_{4}^{\mathrm{e}}$ et le $20^{\mathrm{e}}$ jours de lutte chez les adultes, le $I 7^{\mathrm{e}}$ et $1 \mathrm{e} 23^{\mathrm{e}}$ jours, de lutte chez les antenaises.

- 1'intervalle introduction des béliers vasectomisés- ${ }^{\mathrm{er}}$ pic de fréquence élevée des cestrus est resté pratiquement invariable dans le lot J (- I5), il était beaucoup plus variable dans le lot $\mathrm{J}$ o (tabl. 2), notamment chez les brebis adultes. 

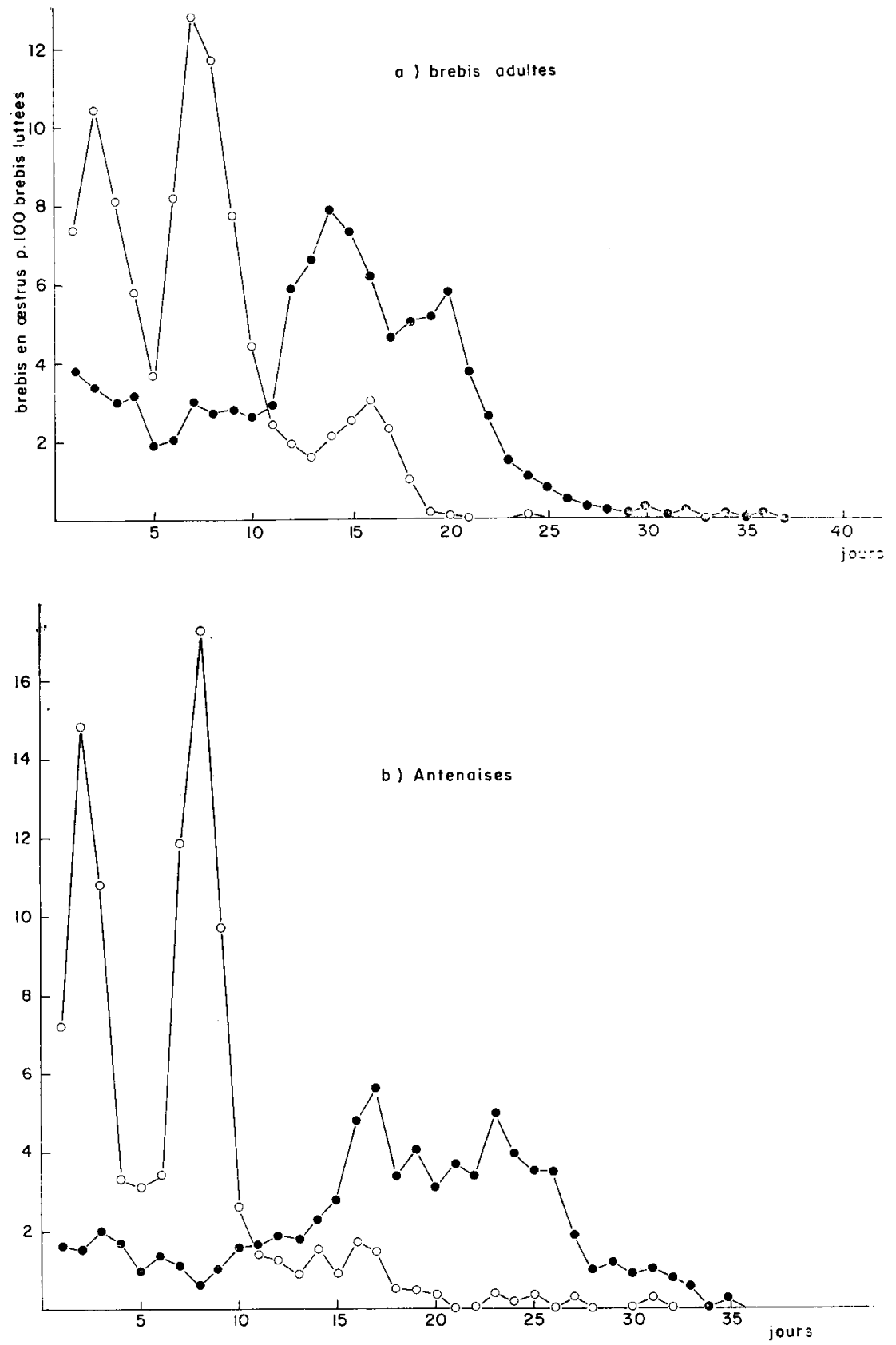

FIG. I. - Courbes des fréquences relatives d'apparition des astrus Lutte de printemps -. - troupeau principal
a) brebis adultes
b) antenaises
$\longrightarrow \operatorname{lot} \mathrm{J}$ o
$\mathrm{O}-\mathrm{O}$ lot $\mathrm{J}-\mathrm{I} 5$

Les béliers vasectomisés sont introduits dans le troupeau ${ }_{5}$ jours avant le début de la lutte (lot (J - I 5)) et le premier jour de la lutte (lot $\mathrm{J}$ o) ; la détection des cestrus commence le premier jour de la lutte. 
La mise en place préalable des béliers vasectomisés a permis d'avancer le jour moyen d'apparition des premiers ruts de ro jours chez les antenaises $(6,4$ au lieu de $\mathbf{I} 6,5$ jours) et de plus de 5 jours chez les adultes (6,7 au lieu de I2). Dans les deux cas, les écarts moyens ont été sensiblement réduits, leurs valeurs respectives étant de 3,42 au lieu de 6,09 jours chez les antenaises, 3,44 au lieu de 4,94 jours chez les adultes; au bout de ro jours de lutte 86,4 p. Ioo des antenaises $(84,7$ à 87,4 p. Ioo) et 79,6 p. Ioo des adultes $(74,4$ à 85,8 p. IOO) étaient saillies au lieu, respectivement, de I4,I p. IOo (3,8 à 30,4 P. IOO) et 26,6 p. IOO (I 3,2 à 43,4 p. IOO).

\section{TABLEAU 2}

Intervalles entre l'introduction des béliers vasectomisés dans le troupeau et les pics de fréquence élevée d'apparition des ostrus

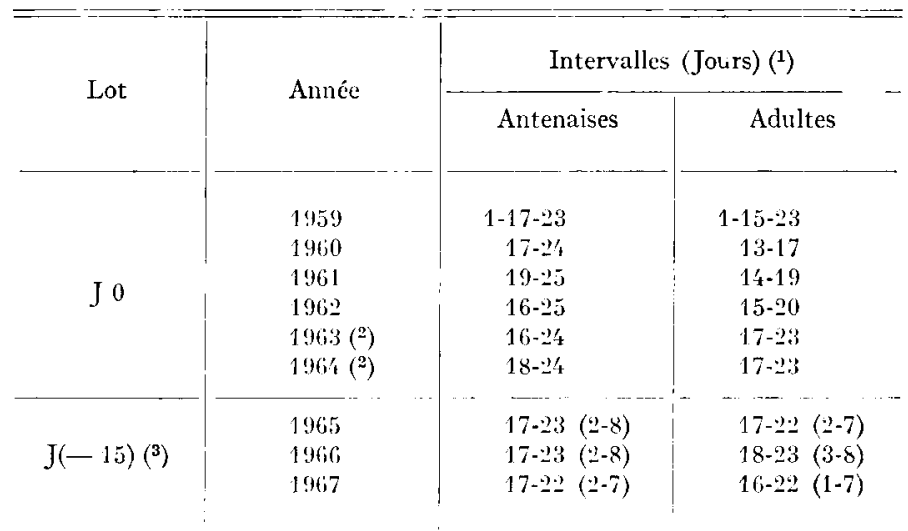

(1) Ces intervalles correspondent aux modes cles courbes de fréquence des cestrus qui ne sont pas normales mais bimodales ou trinodales.

(2) Iin 1963, 5 béliers vasectomisés ont été mêlés aux brebis 9 jours avant le début de la lutte, 10 antres béliers étant introduits le premier jour de la lutte; en 1961,6 béliers vasectomisés ont été mêlés aux brebis 6 jours avant le rébut de la lutte, 10 autres béliers étant introduits le premier jour de la lutte. Les intervalles ont été calculés à partir de la date d'introduction initiale.

(3) Nous indiquons entre parenthèses l'intervalle entre le début de la lutte contrôlé et l'apparition des pies de fréquence élevèe des ostrus.

Iin ce qui concerne les brebis tardonnières, l'écart entre le lot J (- I 5) et le lot J o est encore plus marqué (fig. 2) ; les pics de fréquence maximum des cestrus sont situés respectivement les $8^{\mathrm{e}}$ et $I 8^{\mathrm{e}}$ jours de lutte au lieu des $26^{\mathrm{e}}$ et $35^{\mathrm{e}}$ jours. Le jour moyen d'apparition du premier rut est avancé de Io jours (I2,9 au lieu de 22,7) mais l'écart est légèrement accru (6,I au lieu de 5,I). L'introduction préalable des béliers vasectomisés permet, dans ce lot, la saillie de $37 \mathrm{p}$. Ioo des brebis au cours des Io premiers jours de lutte au lieu d'une moyenne de $5 \mathrm{p}$. Ioo antérieurement.

Précisons, dans ce cas, que la date moyenne de l'agnelage précédant cette lutte n'a pas varié, 8 mars (lot J 0), 7 mars (lot J (- I5); de plus, nous n'avons pas trouvé 
de corrélation significative entre la date de la précédente mise bas et celle de la détection du premier ostrus, les valeurs des coefficients de corrélation entre ces dates étant respectivement :

$$
r_{J_{0}}=+0,04 \text { et } r_{\mathrm{J}}-{ }_{15}=+0,02
$$

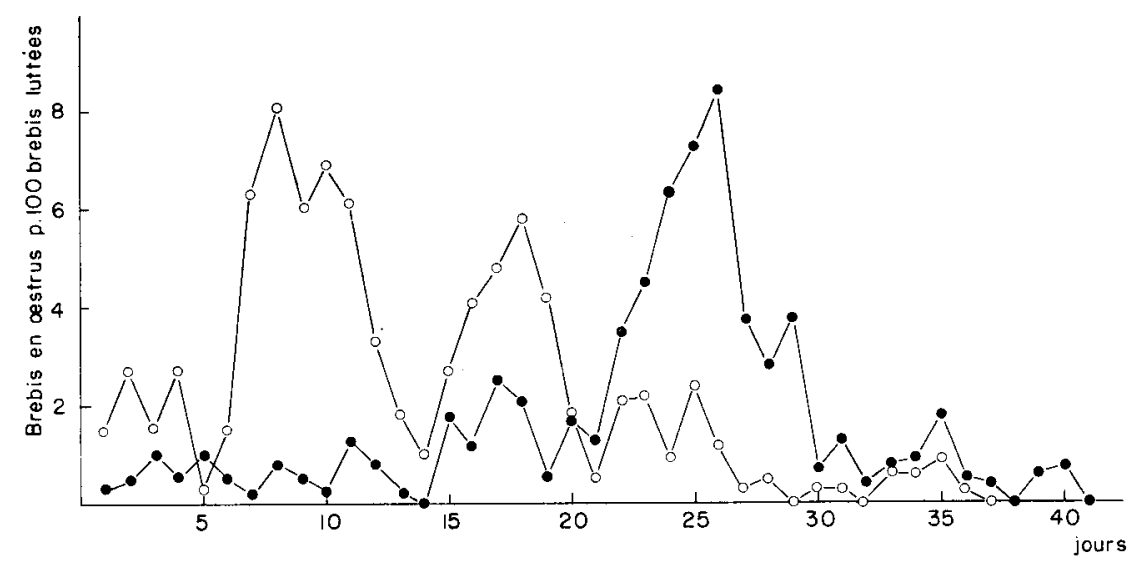

FIG. 2. - Courbes des fréquences relatives d'apparition des premiers cestrus Lutte de printemps - brebis tardonnières

$$
\text { -... lot Jo o- o lot J- I5 }
$$

Les béliers vasectomisés sont introduits dans le troupeau I 5 jours avant le début de la lutte (lot (J - 15)) et le premier jour de la lutte (lot $\mathbf{J}$ o); la détection des oestrus commence le premier jour de la lutte.

\section{B. - Anomalies de détection des ruts}

Lors de 1'étude de la technique de contrôle des ruts utilisée au domaine du Merle, nous avions mis en évidence (PRUD'HON et al., rg66) trois types d'anomalies pouvant correspondre à des erreurs de détection d'œstrus et permettant de fixer la limite supérieure des erreurs commises :

- une brebis, non gestante après saillie au premier cestrus détecté, a un nouvel œstrus moins de I5 jours ou plus de I9 jours plus tard;

- une brebis est détectée par un bélier alors qu'elle est déjà gestante ;

- une brebis, saillie plus de I9 jours avant la fin de la lutte contrôlée, non gestante après cette saillie, n'a pas de nouvel œestrus au cours des I9 derniers jours de lutte.

Les fréquences de ces trois types d'anomalies figurent au tableau 3.

Dans le lot J o, 66 p. roo de ces anomalies sont survenues au cours des I 5 premiers jours de lutte, période pendant laquelle peu de brebis étaient en ostrus chaque jour alors que les mâles, au repos depuis six mois, manifestaient une grande activité. Ces erreurs ont affecté davantage les antenaises que les adultes, soit qu'elles aient été plus attractives pour les béliers, soit qu'elles aient, par inexpérience, accepté plus aisément le chevauchement malgré l'absence d'œstrus.

Dans le lot J (- I5), la fréquence des anomalies du I er et du $3^{\mathrm{e}}$ type a été significativement abaissée chez les antenaises. Compte tenu du grand nombre de femelles en oestrus dès le début des contrôles, les béliers boute-en-train ont sans doute moins cherché à chevaucher celles qui n'étaient pas en rut. 


\section{TABIEAU 3}

Fréquences et pourcentages d'anomalies de détection des astrus en fonction de l'âge des brebis et du moment d'introduction des béliers vasectomisés dans le troupeau

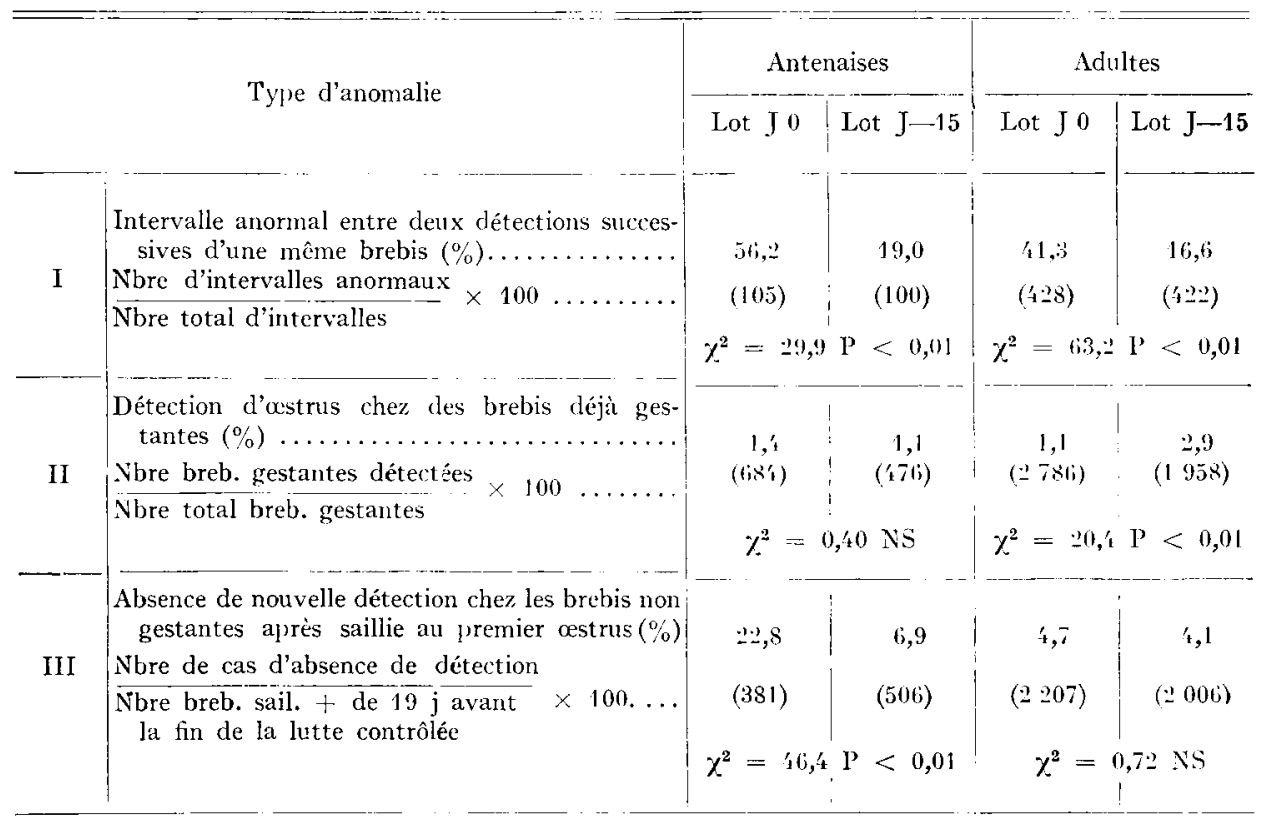

( ) Nombre d'animaux.

I. La durée normale d'un cycle de brebis Mérinos d'Arles est comprise entre 15 et $19 \mathrm{j}$. Lorsque l'intervalle entre 2 détections est infúrieur à 15 j ou supérieur à $19 \mathrm{j}$, il est considérí comme anormal et peut correspondre soit à une anomalie cyclique soit à une erreur de détection. Le pourcentage réel d'erreurs de détection n'est pas calculable puisque l'on ne connaît pas la part des intervalles anormaux qui sont dus à des anonalies cycliques.

II. L'analyse des durées de gestation permet a posteriori de savoir à quel moment la saille fécondante a eu lieu. Il est donc possible après mise bas d'évaluer le pourcentagre cle brebis ayant śté marquées par cles béliers alors qu'elles étaient déjà gestantes.

III. Nous comparons ici les brebis saillies plis de 19 jours avant la fin de la lutte, non ressaillies pendant le reste et r'ayant pas mis bas, à l'ensemble des brebis saillies jendant la même période. 
Chez les brebis adultes, le nombre des anomalies du premier type a baissé mais les anomalies du second type ont été plus fréquentes $(\mathrm{P}<0,0 \mathrm{I})$ ce qui est normal puisque l'avancement de l'apparition des premiers oestrus dans ce lot diminue la période de séjour des béliers vasectomisés avec des brebis non gestantes et augmente la durée de leur séjour avec des brebis gestantes.

Au total, la modification de technique de lutte a entraîné une amélioration sensible de la qualité de détection des œstrus des antenaises, une amélioration seulement légère dans le cas des brebis adultes.

\section{C. - Fertilité des brebis}

Au tableau + figurent les valeurs des paramètres de la fertilité des antenaises, des brebis adultes et des tardonnières après les 30 premiers jours de lutte.

\section{TABLEAU 4}

Valeurs des différents paramètres de la fertilité des brebis soumises à une lutte de printemps de 30 jours en fonction de l'áge des brebis et du moment d'introduction des béliers vasectomisés dans le troupeau

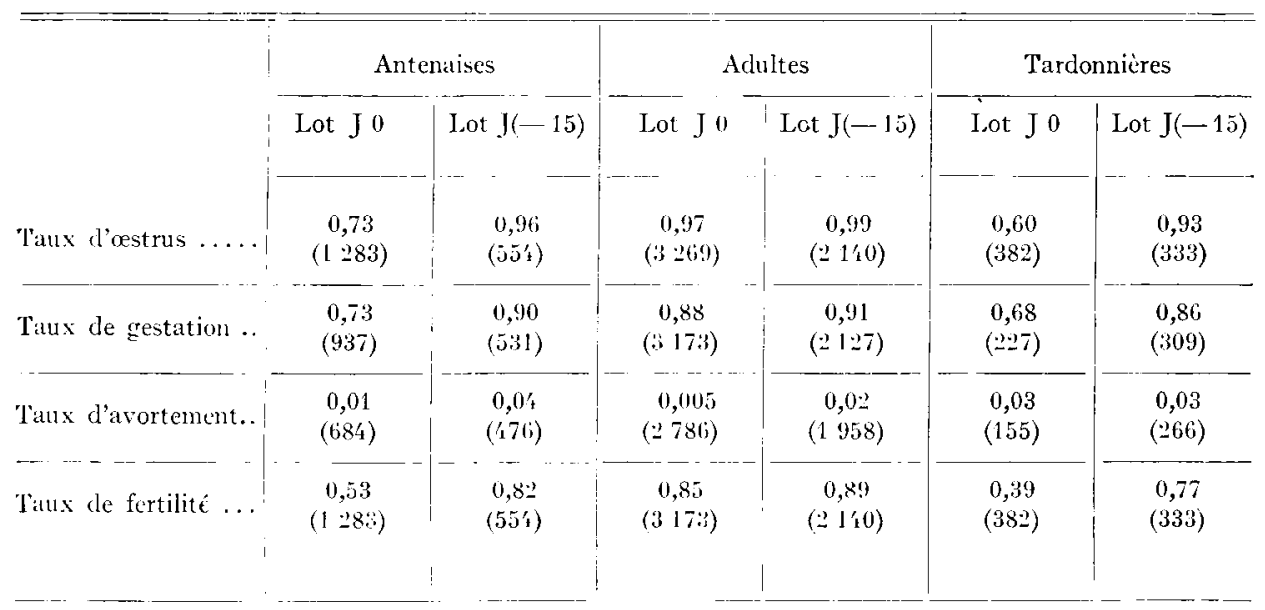

( ) Nombre d'animaux. la lutte.

Taux d'astru's : Xombre moyen de brebis entrant en astrus au cours de la lutte par brebis présente à

Taux de gestation : Nombre moyen de brebis gestantes par brebis saillie.

T'aux d'avortement : Nombre moyen de brebis avortant par brebis gestante.

Taux de fertilitú : Nombre moyen de brebis mettant 1 as par brebis présente à la lutte.

Il est possible de constater que si le fait d'avancer de i5 jours la date d'introduction des béliers vasectomisés n'améliore pas de façon très sensible la fertilité des brebis adultes, il n'en est pas de même pour les antenaises dont le taux de fertilité s'élève de 0,53 à 0,82 et pour les tardonnières oì il passe de 0,39 à 0,77 . I)ans ces deux cas, les taux d'œestrus et de gestation sont sensiblement accrus. 


\section{DISCUSSION}

Ces résultats montrent tout d'abord que :

- au printemps l'activité sexuelle des brebis Mérinos d'Arles a été influencée chaque année par l'introduction de béliers vasectomisés dans le troupeau.

Cet effet psychique s'est produit aussi bien avec introduction des béliers le 25 avril que le Io mai : les cestrus des brebis non allaitantes se sont distribués selon une courbe de fréquence à 2 pies très marqués espacés de 6 à 7 jours, phénomène signalé également par Schinckei, (I954 a), EYAL (I958), DAUZIER et I)ENOY (I964), PRUD'Hon et al. (Ig66).

- Il semble cependant que la date d'introduction des béliers vasectomisés ait eu une influence sur 1'amplitude du groupage des chaleurs et sur l'importance relative des pics.

Nous avons constaté trois années de suite qu'une proportion très significativement supérieure des brebis, notamment des antenaises, entraient en œestrus au moment des pies lorsque l'introduction des béliers avait lieu le 25 avril et non le to mai ; bien que ces comparaisons n'aient pas été faites la même année, il est peu probable, compte tenu de la répétabilité de ce phénomène, qu'il s'agisse d'un effet lié à l'année.

De même dans le lot J (- I5), l'amplitude du $2^{\mathrm{e}}$ pic s'est révélée supérieure ou égale à celle du premier pic alors qu'elle était inférieure dans le lot J o.

Selon une hypothèse de SchINcker, (r954a), la présence de mâles parmi les femelles en fin d'anœstrus saisonnier aurait pour effet de déclencher l'ovulation silencieuse de la majeure partie d'entre elles au cours des six jours suivant leur introduction. Le premier œstrus décelable apparaîtrait un cycle plus tard. Dans cette hypothèse, il est normal qu'une introduction plus précoce des béliers dans le troupeau, survenant à une époque où un plus grand nombre de femelles n'ont pas repris une activité sexuelle régulière se traduise par une augmentation de l'amplitude relative des pics et une amélioration du groupage des chaleurs.

Il est plus difficile d'expliquer la variation d'importance relative des deux pics, puisque, à notre connaissance, leur origine n'est pas encore connue. Schinckei. (I954 b) pensait qu'après une période d'intense activité les béliers détecteurs avaient une baisse temporaire de libido ce qui aurait entrainé l'existence de ces deux pics. Dans ce cas, on devrait observer un troisième pic 20 jours environ après l'apparition du premier. Nous ne l'avons pas observé. On peut penser que l'introduction des béliers déclenche très rapidement l'ovulation chez les brebis ayant déjà des follicules en cours de développement alors que les autres brebis ont besoin de plusieurs jours avant d'en arriver au même stade; autrement dit, 1'importance respective des deux pics refléterait la proportion respective de femelles ayant déjà un début d'activité sexuelle ou étant encore ua repos sexuel.

- Il est intéressant de noter que chez les antenaises des deux lots et chez les brebis adultes en contact avec les béliers vasectomisés plusieurs jours avant la lutte contrôlée (lot J (- I5) et années Ig63 et I964 du lot J o, les pics apparaissent très régulièrement I7 et 23-24 jours après l'introduction des béliers vasectomisés dans le 
troupeau. Au contraire, chez les brebis adultes mises en contact avec les béliers vasectomisés le premier jour de lutte, les premiers pics apparaissent I3 à I5 jours plus tard. Ce résultat, en contradiction avec 1'hypothèse de SchisckeI (I954 a), pourrait être lié à la manière dont est organisée la lutte : chaque année les cases des béliers de lutte sont édifiées dans la bergerie 3 ou 4 jours avant le début des contrôles; elles ont toujours le même aspect et le même emplacement ; elles restent vides jusqu'au premier jour de lutte. On peut se demander si la vue des cases ou éventuellement l'odeur des béliers ayant pu subsister sur les claies depuis l'automne ne pourrait suffir à déclencher l'activité sexuelle des brebis adultes. WASTON et RADFORD (I960) ont déjà montré que ni le contact avec les béliers, ni même leur vue ne sont indispensables au déclenchement psychique de l'activité sexuelle des brebis. Le stimulus n'aurait pas d'action sur les antenaises qui n'ont jamais vu ces cases de lutte antérieurement. Il n'aurait plus d'action sur les adultes lorsque l'introduction des béliers vasectomisés précède l'édification des cases.

Le cas des tardonnières est sensiblement différent : au moment où commence la lutte, ces femelles, qui ont agnelé en moyenne le 7 mars, allaitent leurs agneaux respectivement depuis $50 \pm$ II 8 jours ( $\operatorname{lot} \mathrm{J}\left(-\mathrm{I}_{5}\right)$ ) et $65 \pm \mathrm{I}_{3}, 3$ jours (lot J o). A l'effet de l'ancestrus saisonnier, doit donc s'ajouter, pour elles, celui, plus ou moins marqué, de l'ancestrus de lactation. Ceci se traduit par une allure différente de la courbe d'apparition des ostrus. Ainsi le premier pic des tardonnières coïncide sensiblement avec le deuxième pic des autres brebis, ce qui semble bien l'indice d'un repos sexuel plus profond ; par ailleurs, il existe dans le lot J ( - I5) un deuxième pic très net, situé 35 jours après la mise en place des béliers, ce qui laisse supposer chez les femelles en œestrus à ce moment-là, l'existence préalable de deux ovulations silencieuses.

MaUlíon et I)AUziér (I965) ont constaté à l'automne et en race Ile-de-France que la durée de l'intervalle moyen mise bas-premier ostrus des femelles allaitantes était légèrement abrégée en présence de béliers vasectomisés. Nos résultats semblent montrer qu'au printemps les brebis Mérinos d'Arles allaitantes depuis 50 jours reprennent pour la plupart une activité sexuelle régulière à la suite de l'introduction de béliers boute-en-train parmi elles. Cette reprise paraît liée surtout à l'effet de la présence des mâles, elle ne semble pas dépendre, dans le cadre de nos observations, de la date d'introduction des béliers ni de la date de la précédente mise bas.

- La présence de béliers vasectomisés dans le troupeau, 15 jours avant le début des contrôles d'œestrus, a réduit sensiblement le pourcentage d'anomalies de détection des oestrus. La réduction du nombre d'erreurs véritables est sans doute plus importante encore puisqu'un certain nombre d'anomalies ne correspondent pas à des erreurs et doivent avoir une fréquence sensiblement constante : c'est ainsi que des cas d'œestrus chez des brebis gestantes ont été constatés par Wir.LIAMs et al. (I956), HUxTER (I964), des cas de superfœetation indiscutables ont même été signalés par Rominson (I95I) et MatTer (I965). Par ailleurs, Dolling et Nicolson ig67 ont constaté que 4,3 p. Ioo des brebis Mérinos présumées gestantes en l'absence d'un nouvel oestrus au cours des 20 jours suivant la saillie n'agnelaient pas. Ils ont confirmé par une série d'autopsies que ce phénomène pouvait être attribué essentiellement à des cas de mortalité embryonnaire.

- Il faut souligner enfin l'intérêt pratique et économique de l'introduction préalable des béliers vasectomisés dans le troupeau. I)ans le lot $\mathrm{J}$ (- I5) plus de 
80 p. Ioo des brebis ont été saillies au cours des ro premiers jours de lutte au lieu de I5 à 30 p. roo dans le lot $\mathrm{J}$ o ; ceci a permis à la quasi-totalité des femelles non gestantes après la première saillie d'être à nouveau accouplées. Le nombre des brebis ayant agnelé à l'automne a été accru de I3 p. Ioo et la date moyenne d'agnelage avancée de 8 jours environ ce qui, compte tenu de l'évolution du marché de l'agneau en fin d'année, est un avantage appréciable. Un résultat analogue pourrait sans doute être obtenu avec un séjour préalable des béliers boute-en-train moins prolongé. OvERJA et al. (Ig66) ont réalisé un groupage des chaleurs semblable au nôtre en introduisant, I6 jours avant le début de la lutte de printemps, des béliers vasectomisés qu'ils laissaient en place 8 jours seulement.

\title{
Reçu pour publication en février 1969.
}

\section{SUMMARY}

\author{
EFFECTS ON THE ONSET OF CESTRUS, FREQUENCY OF ERROR IN THE DETECTION \\ OF HEATS AND EWE FERTILITY WHEN VASECTOMIZED RAMS ARE INTRODUCED INTO A FLOCK \\ OF " MÉRINOS D'ARLES " FIFTEEN DAYS BEFORE THE BEGINNING OF SPRING MATING
}

Between 1959 and I964, 4934 Mérinos d'Arles ewes (382 of which were suckling) were submitted to a spring mating beginning May i 2 t 2 days, and lasting $30-35$ days. The published results (PRUD'HON et al., I 966) showed the effect of the presence of males on the release of female sexual activity. Between 1965 and 1967,3027 ewes (333 of which were suckling) were submitted to spring mating at the same date and for the same length of time. Analogous methods were used, but i 5 days before the controlled mating, vasectomized rams were introduced into the flock at the ratio of one for $80-100$ ewes. The lots of ewes will be respectively referred to as lots $\mathrm{J}$ o and $\mathrm{J}-\mathrm{I} 5$.

Results were compared on the onset of estrus, the frequency of error in the detection of heats, and flock fertility parameters.

In all the non-suckling ewes, the first heat frequency curve shows 2 peaks, spaced at 5-6 days, generally occuring $17-22$ or 23 days after the introduction of the rams. However, in the adult ewes of lot $\mathrm{J}$ o, peaks are found between days $\mathrm{r}_{4}$ and 20 of mating. It is postlated that the construction of mating stalls acted as a stimulus.

The mean date of the onset of estrus is advanced 9.8 days for the 2-tooths ewes of lot J-I5, and 5.6 days for the adults of the same lot. The grouping of heats is itself ameliorated, the standard deviation passing respectively from 6.09 days (lot $\mathrm{J}$ o) to 3.42 days (lot $\mathrm{J}-\mathrm{I}_{5}$ ) in the 2-tooths ewes, and from 4.94 days (lot J - I 5) to 3.44 days (lot $\mathrm{J}$ o) in the adults.

In suckling ewes, the principal peak of the estrus frequency curve coincides with the second peak in the other ewes. In lot $\mathrm{J}-\mathrm{I} 5$, there is a second peak 35 days (about 2 cycles) after the introduction of teasers. The date of onset of the first estrus is correlated solely to that of the introduction of males, and not to the date of parturition.

Frequency in error of heat detection during controlled mating is much lower in lot J - I5 than in lot $\mathrm{J} \mathrm{o}$. Reduction in the percentage of errors is especially noticeable in the 2 -tooths ewes.

The fertility rate of ewes after 30 days of mating rises from 0.53 to 0.82 in the 2 -tooths ewes of lots J o and J - I 5 , from 0.85 to 0.89 in adults, and from 0.39 to 0.77 in suckling ewes. These results are due to an increase in the number of females in estrus during controlled mating, and the possibility offered to more than $80 \mathrm{p}$. I oo of the females of lot $\mathrm{J}-$ I 5 to mate again, if they were not pregnant after the first mating.

\section{RÉFÉRENCES BIBLIOGRAPHIQUES}

Dauzier L., Denoy I., I964. Quelques résultats de la lutte des brebis du troupeau Mérinos d'Arles du domaine du Merle. Journées C. E. T. A., Étude no 963 .

Dolling C. H. S., Nicolson A. D., 1967. Vital statistics for an experimental flock of Merino sheep. IV. Failure in conception and embryonic loss as cause of failure to lamb. Aust. J. agric. Res., 18, $767-788$. 
Edgar D. G., Bilkey D. A., 1963. Advancing the breeding season in ewes. Proc. Ruakura Fmrs' Conf. Week., 6-I3.

EyaL E., I958. The introduction of rams as a factor influencing sexual activity of ewes. Kiavim, 8, 359366.

Hughes R. L., I964. Effect of changing cages, introduction of the male and other procedures on the ostrous cycle of the rat. C. S. I. R. O. Wild. Res., 9, I 5 .

Hunter G. L., I964. The effects of season and mating on cestrus and fertility in the ewe. Proc. S. Afr. Soc. Anim. Prod., 3, 195-206.

Lamond D. R., 1959. Effect of stimulation derived from other animals of the same species on œestrous cycles in mice. J. Endocr., 18, 343. Cité par RadFoRd, I966.

Lishman A. W., Hunter G. L., I966. Sexual activity in the ewe following isolation from the ram during the breeding season. S. Afr. J. agric. Sci., 9, 993-1004.

Matter H. E., 1965. A case of superfotation in the Karakul sheep. Berl. Münch. tierärztl. Wschr., 78, $469-47^{\circ}$. in Anim. Breed. Absir., 35, 467.

Mauléon P., Dauzier L., I965. Variations de durée de l'anostrus de lactation chez les brebis de race Ile-de-France. Ann. Biol. anim. Biochim. Biophys., 5, I31-I43.

Overja A. A., Whiteman J. V., Gould M. B., Thrift F. A., ig66. Effect of teasing on mating and lambing performance of spring bred ewes. Misc. Publs. Okia. agric. Exp. Stn., 78, 34*39. in Anim. Breed. Absir., 35, 476.

Prud'hon M., Denoy I., Dauzier L., Desvignes A., I966. Étude des résultats de six années d'élevage des brebis Mérinos d'Arles du domaine du Merle. I. Le contrôle des ruts et sa validité. Ann. Zootech., 15, 123 -I33.

Prud'hon M., Denoy I., Desvignes A., Golssopoulos J., ig68. Étude des résultats de six années d'élevage des brebis Mérinos d'Arles du domaine du Merle. II. Relations entre l'áge, le poids, l'époque de lutte des brebis et les divers paramètres de la fécondité. Ann. Zootech., 17, 31-45.

Radford H. M., I966. Regulation of the breeding season in mammals. Proc. Aust. Soc. Anim. Prod., 6, I9-3I.

Riches J. M., WATSON R. M., i954. The influence of the introduction of rams on the incidence of oestrus in Merino ewes. Aust. J. agric. Res., 5, I4I-I47.

RoBinson T. J., I95I. Reproduction in the ewe. Biol. Rev., 26, 121-J57.

Schinckel P. G., I954a. The effect of the presence of the ram on the ovarian activity of the ewe. Aust. J. agric. Res., 5, 465-469.

SCHINCKEL P. G., I954 $b$. The effect of the ram on the incidence and occurrence of oestrus in ewes. Aust. itet. J., 30, 189-195.

SHELTON M., ig6o. Influence of the presence of a male goat on the initiation of ostrus cycling and ovulation in angora does. J. Anim. Sci., 19, 368-375.

Sinclair A. N., I950. A note on effect of the presence of rams on the incidence and occurrence of cestrus in ewes. Aust. vet. J., 26, 37-39.

Underwood E. J., Shier F. I., Davenport N., I944. Studies in sheep husbandry in Western Australia. $V$. The breeding season of Merino, crossbred and Brilish breed ewes in the agricultural districts. J. Dep. Agric. West. Aust., 21, I35-143.

Watson R. M., Radford H. M., I960. The influence of rams on onset of ostrus in Merino ewes in the spring. Aust. J. agric. Res., 11, 65-7 1 .

Whitten W. K., 1958. Modification of the cycle of the mouse by external stimuli associated with the male. J. Endocr., 17, 307-313.

Williams S. M., Garrigus U. S., Norton M. W., Nalbandov A. V., i956. The occurrence of oestrus in pregnant ewes. J. Anim. Sci., 15, $978-983$. 